\title{
Environmental Factors Contribute to the Transmissibility of COVID-19: Evidence from an Improved SEIR Model
}

Jie Li

Beijing Normal University

Kun Jia ( $\nabla$ jiakun@bnu.edu.cn )

Beijing Normal University

Wenwu Zhao

Beijing Normal University

Bo Yuan

Beijing Normal University

Yanxu Liu

Beijing Normal University

\section{Research Article}

Keywords: COVID-19, Brazilian states, Environmental factors, Improved SEIR model

Posted Date: May 11th, 2021

DOI: https://doi.org/10.21203/rs.3.rs-495262/v1

License: (c) (1) This work is licensed under a Creative Commons Attribution 4.0 International License. Read Full License 


\title{
Environmental factors contribute to the transmissibility of
}

\section{COVID-19: Evidence from an improved SEIR model}

\author{
Jie Li ${ }^{1}$, Kun Jia ${ }^{1, *}$, Wenwu Zhao ${ }^{2,3}$, Bo Yuan ${ }^{1}$, Yanxu Liu ${ }^{2,3}$ \\ 1 State Key Laboratory of Remote Sensing Science, Faculty of Geographical Science, Beijing Normal University,
}

Beijing 100875, China

2 Stake Key Laboratory of Earth Surface Processes and Resource Ecology, Faculty of Geographical Science,

Beijing Normal University, Beijing 100875, China

3 Institute of Land Surface System and Sustainable Development, Faculty of Geographical Science, Beijing

Normal University, Beijing 100875, China

* Correspondence: jiakun@bnu.edu.cn; Tel.: +86-010-5880-0152 


\section{ABSTRACT}

COVID-19 is ravaging Brazil, and its spread shows spatial heterogeneity. Changes in the environment have been implicated as potential factors involved in COVID-19 transmission. However, considerable research efforts have not elucidated the risk of environmental factors on COVID-19 transmission from the perspective of infectious disease dynamics. The aim of this study is to model the influence of the environment on COVID-19 transmission and to analyze how the socio-ecological factors affecting the probability of virus transmission in 10 states dramatically shifted during the early stages of the epidemic in Brazil. First, this study used a Pearson correlation to analyze the interconnection between COVID-19 morbidity and socio-ecological factors, and identified factors with significant correlations as the dominant factors affecting COVID-19 transmission. Then, the time-lag effect of dominant factors on the morbidity of COVID-19 was investigated by constructing a distributed lag nonlinear model, and the results were considered to be an improvement over the SEIR model. Lastly, a machine learning method was introduced to explore the nonlinear relationship between the environmental propagation probability and socio-ecological factors. By analyzing the impact of environmental factors on virus transmission, it can be found that population mobility directly caused by human activities had a greater impact on virus transmission than temperature and humidity. The heterogeneity of meteorological factors can be accounted for by the diverse climate patterns in Brazil. The improved 
SEIR model was adopted to explore the interconnection of COVID-19 transmission and the environment, which revealed a new strategy to probe the causal links between them. Keywords: COVID-19; Brazilian states; Environmental factors; Improved SEIR model 


\section{Introduction}

As the deadly wave of COVID-19 epidemic began to sweep across the world, the first case of COVID-19 in Brazil was reported on 25 February 2020 in São Paulo[1]. The World Health Organization (WHO) officially declared COVID-19 a pandemic in March 2020. Most countries, including Brazil, have implemented widespread social distancing restrictions to mitigate the spread of the virus, which indicates that these strategies can effectively reduce the number of cases and associated deaths[2]. Depending on the real-time statistics released by Johns Hopkins University, Brazil had approximately 7.676 million confirmed cases by the end of 2020, ranking third after the United States and India; thus, it was one of the most affected countries in the world.

In Brazil, a country of continental dimensions noted for its enormous socio-economic and environmental diversity, the spread of COVID-19 is very heterogeneous, affecting cities and rural regions differently[3]. As the primary component of the socioecological system, nonlinear complex global climate change presents a threat to human health in a variety of ways in relation to continuous COVID-19 transmission[4-7]. Recent studies have shown that the spread of COVID-19 is thought to be greater in cold and temperate climates and that it spreads slowly in warm, humid environments[8-10]. In addition, large-scale and diffuse human migration can affect the geographical distribution of infections and the spreading pattern of the pandemic[11], and COVID19 transmission significantly decreased with mobility controls[12]. The social and natural environment has a superposition effect on COVID-19 transmission in relation 
to COVID-19 epidemiology. However, numerous studies so far have analyzed the interconnection between the environment and COVID-19 transmission through statistical analysis methods. The potential association has only been explored on a superficial level, resulting in different conclusions regarding the driving effects of environmental factors on COVID-19 transmission in the same area.

To date, a handful of studies have analyzed, made predictions about and evaluated the COVID-19 pandemic by modifying classical epidemiological dynamic models, including the SIR, SEIR, and SEIRD methods[13-17]. These studies combined the characteristics of COVID-19 and medical prevention and control measures to establish a dynamic model of infectious diseases in line with regional features to analyze and explore the trend of development of the pandemic. Although these epidemiological models are useful for estimating the dynamic process of COVID-19 transmission, they are primarily focused on the impact of social behavior on COVID-19 transmission, especially social distancing and quarantines, and few studies have considered the impact of meteorological factors on the transmission of COVID-19. It should be noted that it is also possible to comprehensively consider the influence of socio-ecological factors on COVID-19 transmission in the model, which can systematically explore the mechanism of environmental factors on COVID-19 transmission from the dynamic process of virus transmission.

Therefore, this study was intended to explore the role of the environment in COVID19 transmission by improving the SEIR model, in which socio-ecological factors and 
active cases for the 10 seriously affected states of Brazil can be established. First, Pearson correlation was used to screen environmental factors that significantly affected the pandemic trends to identify which ones were dominant. Then, the time-lag effect of environmental factors on the spread of COVID-19 was analyzed. Considering the hysteresis of environmental factors, the classical SEIR infectious disease model was improved, and the nonlinear relationship between socio-ecological factors and COVID19 transmission was built by adopting the machine learning method to explore the impact of environmental change on COVID-19 transmission. In this study, environmental factors were added to the improved SEIR model as a process parameter, which was named the environmental propagation probability, and the least square method was used to fit the trends of the simulated cases to the active cases. Accordingly, the environmental propagation probability was optimized by using the associated optimization algorithm to complete the above procedure. Therefore, the impact of socio-ecological factors on virus transmission was analyzed from the perspective of infectious disease dynamics, and the role and importance of environmental changes on COVID-19 transmission were also determined. This study provides a reference to guide and scientific evidence to formulate COVID-19 prevention and control policies from the perspective of environmental change. 


\section{Materials and methods}

\subsection{Study area}

The Brazilian government has offered a column of COVID-19 to report the cases in real-time. In this study, 10 severely affected states were selected from among the 27 states of Brazil, namely, Amazonas, Bahia, Ceará, Maranhão, Minas Gerais, Pará, Pernambuco, Rio de Janeiro, São Paulo, and Distrito Federal. The study area includes various climate types (Fig. 1, adapted from Alvares et al[18]), covering longitudes from $34^{\circ} 48^{\prime} 24^{\prime \prime} \mathrm{W}$ to $73^{\circ} 47^{\prime} 44^{\prime \prime} \mathrm{W}$ and latitudes from $2^{\circ} 37^{\prime} 56^{\prime \prime} \mathrm{N}$ to $25^{\circ} 18^{\prime} 12^{\prime \prime} \mathrm{S}$. Fig. 2 shows the COVID-19 data, including daily newly confirmed cases and active cases in the 10 Brazilian states.

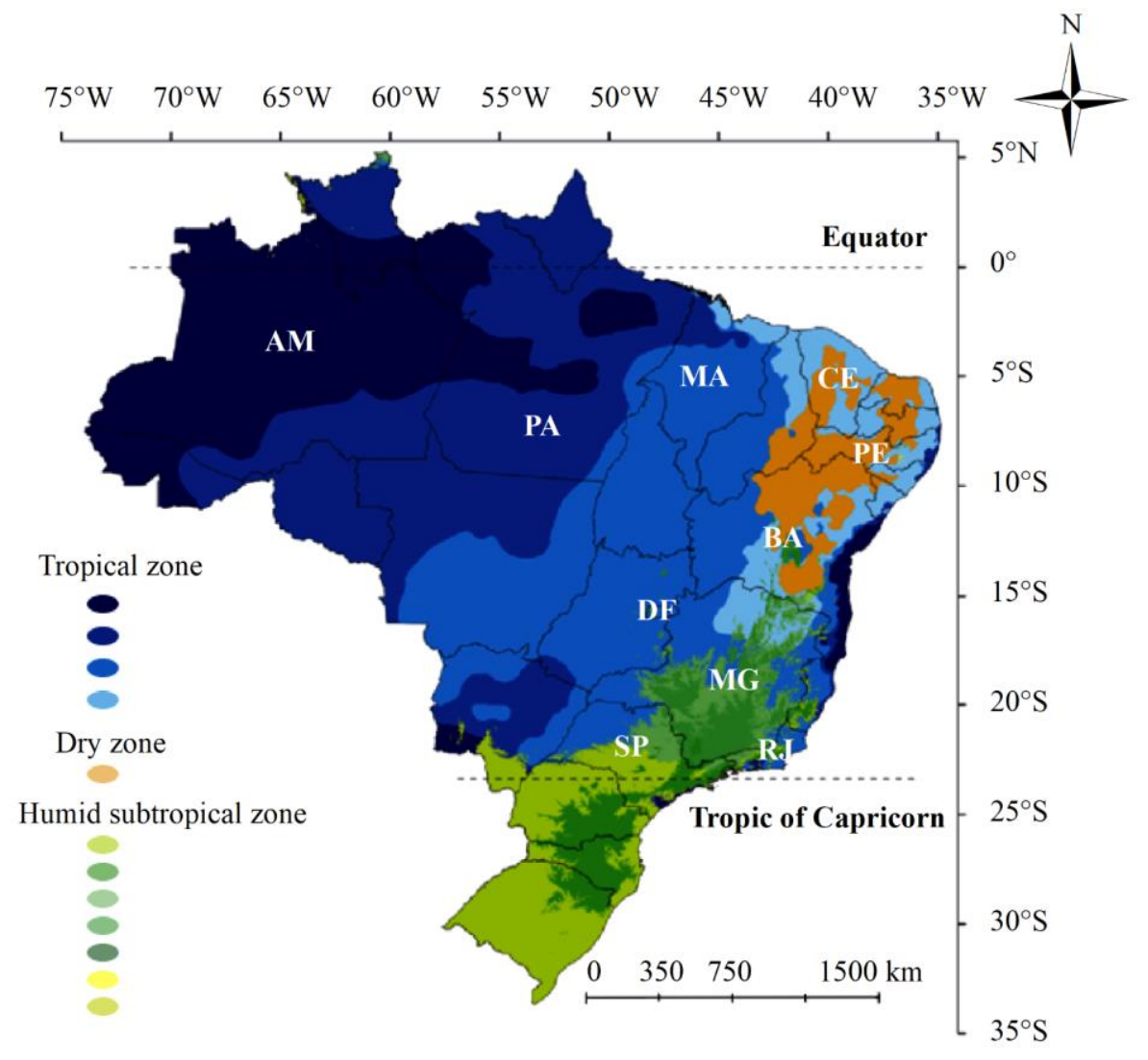

Fig. 1 The location of Brazil with its Köppen climate types. 


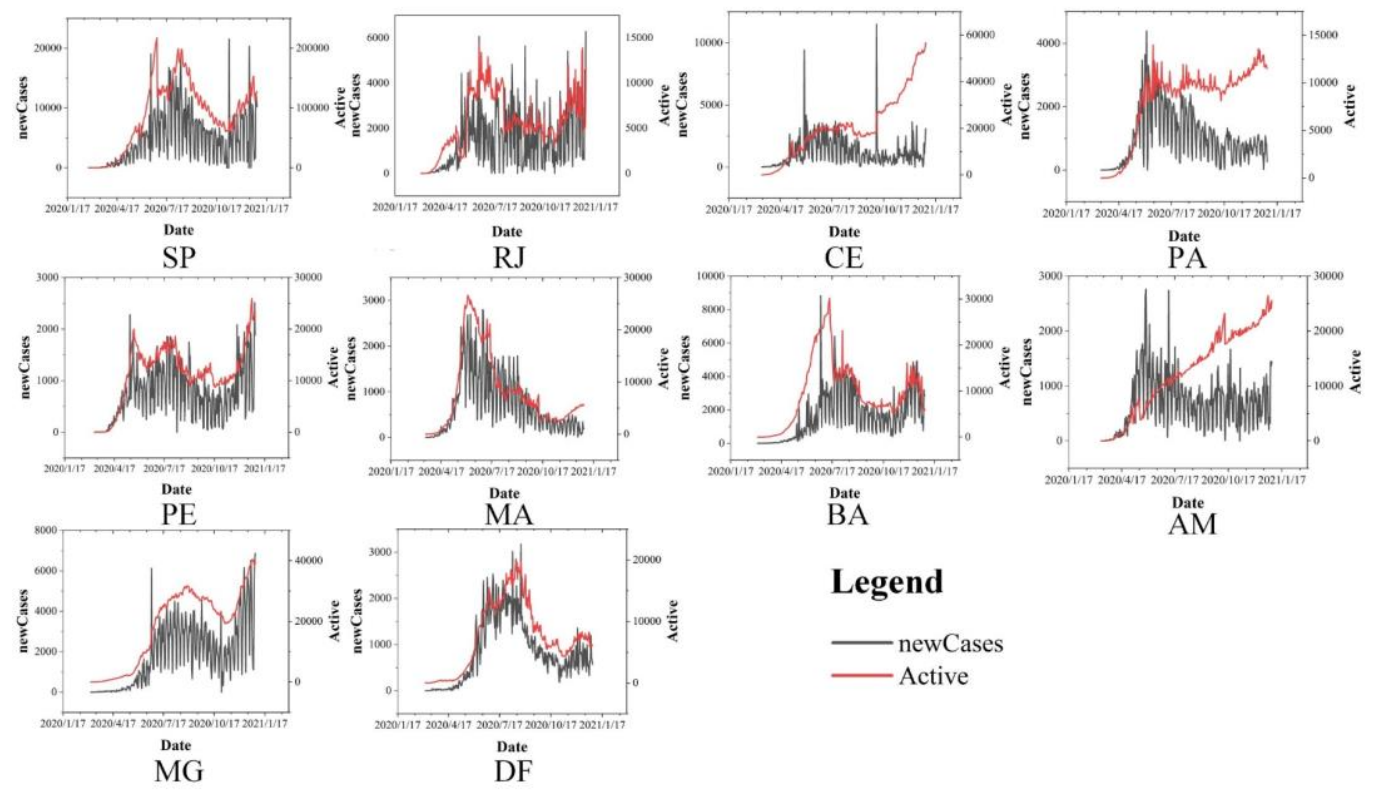

Fig. 2 COVID-19 data from 10 states in Brazil.

\subsection{Data collection}

The COVID-19 dataset includes the daily number of cumulative confirmed cases, cumulative deaths, and cumulative cured cases of COVID-19 in the 10 states, which were officially reported by the Ministry of Health of Brazil from February 25 to December 31, 2020. This study concentrated on the interior of the Brazilian states. Meteorological data were collected from the ERA5 reanalysis dataset (https://cds.climate.copernicus.eu), which were further processed to the state level using zonal statistical methods in ArcGIS10.2. The population mobility was collected from the Google Map Community Mobility Reports (https://www.google.com/covid19/mobility/), which are designed to provide insights into what has changed in response to policies aimed at combating COVID-19. The reports describe movement trends over time by geography across different categories 
of places, such as retail and recreation, groceries and pharmacies, parks, transit stations, workplaces, and residential areas.

\subsection{Methods}

In this study, the Pearson correlation was used to screen environmental factors, and those with a significant correlation between the environment and morbidity were identified as the dominant environmental factors affecting COVID-19 transmission. The distributed lag nonlinear model was used to explore the hysteresis effect of environmental factors on COVID-19 propagation, and the results were used as a reference during the modelling. Notably, this study was focused on the establishment of the improved SEIR model, and using the model to optimize the environmental propagation probability under the condition of simulation cases can fit the active cases well. Then, a machine learning method was used to build the nonlinear relationship between environmental factors and environmental propagation probability. According to the results, the role of environmental factors in COVID-19 transmission was analyzed and discussed.

Pearson correlation analysis was applied to explore the interconnection between the socio-ecological factors and morbidity from COVID-19. Significant correlations were considered at $\mathrm{P}<0.01$. Environmental factors that were significantly correlated with morbidity and had a weak correlation among environmental factors were selected as the dominant environmental factors affecting COVID-19 transmission to be subjected subsequent analysis. 
The distributed lag nonlinear model (DLNM) was first introduced by Gasparrini and Armstrong in 2010 to explore the influence of meteorological factors on human health, specifically assessing the air temperature effect on health[19]. The central idea is a cross basis function. By selecting the appropriate basis function for the exposure-response and exposure-hysteresis dimensions, the cross-basis function is obtained by calculating the tension product of the two basic functions, and then the cross basis is included in the model for analysis. The DLNM has been used to provide a means to assess the nonlinear and delayed effects as well as the relative risks (RR) of different environmental factors within the lag period[20-22]. In this study, the DLNM was used to identify the lag period of environmental factors on COVID-19 transmission and analyze the impact of socio-ecological factors on human health under different lag periods. The results of the DLNM are the basis for the modelling. The dlnm and splines packages in R software (version 3.6.3) were used to construct the model. The resulting model is

$$
\log \left[E\left(y_{t}\right)\right\rceil=\alpha+\sum c b(E, d f, \operatorname{lag}, d f)+n s(\text { time }, d f)
$$

where $t$ is the observation day; $E(y t)$ is the expected value of the observed incidence of COVID-19 on day $t ; \alpha$ is the intercept; and $c b$ is a cross-basis matrix used to estimate the nonlinear relationship between environmental factors and COVID-19 incidence and describe the lag effects of the factors. On the cross basis, $E$ is the environmental factor with 2 degrees of freedom $(d f)$, the lag is up to 15 days, and time is the indicator variable constructed using the natural spline to control long-term trends. 
The classical SEIR model in epidemiology is used to assess the spread of infection and divides the population into susceptible $(S)$, exposed $(E)$, infectious $(I)$ and recovered $(R)$ populations, in which only infected people are considered to be infectious[23]. Since COVID-19 first appeared, it was found to be highly contagious, the spread of COVID-19 has its own specific characteristics: there are symptomatic and asymptomatic infected people who are infectious, and infectious people in the incubation period are contagious and can spread the virus further among people before they experience some or all of these symptoms[24, 25]. Therefore, compared with the classical SEIR model, the improved SEIR model considered the transmission risk of patients in quarantine, and the impact of prevention and control measures such as quarantine and environmental factors on the spread of COVID-19 were also considered.

Based on the classical SEIR model, this study takes quarantine measures to prevent infectious diseases into account, adding quarantined susceptibility $\left(S_{q}\right)$, quarantined incubation $\left(E_{q}\right)$ and quarantined infection $\left(I_{q}\right)$ to the model; it also considers asymptomatic infection $\left(I_{A}\right)$ and symptomatic infection $\left(I_{S}\right)$. In addition, since the effect of treatment varies from person to person and the probability of recovery is different with different levels of the disease, the stock of associated deaths $(D)$ was added to the improved SEIR model. The COVID-19 dynamics (Fig. 3) are modelled by the following equation system:

$$
\begin{array}{r}
\frac{d S}{d t}=-\frac{(1-\rho) \varphi\left(\beta_{A} \varepsilon E+\beta_{S} \varepsilon E+\beta_{A} I_{A}+\beta_{S} I_{S}\right) S}{N} \\
-\frac{\rho \varphi\left(\beta_{A} \varepsilon E+\beta_{S} \varepsilon E+\beta_{A} I_{A}+\beta_{S} I_{S}\right) S}{N}+\omega S_{q}
\end{array}
$$




$$
\begin{gathered}
\frac{d E}{d t}=\frac{(1-\rho) \varphi\left(\beta_{A} \varepsilon E+\beta_{S} \varepsilon E+\beta_{A} I_{A}+\beta_{S} I_{S}\right) S}{N}-\alpha(1-\delta) E-\alpha \delta E \\
\frac{d I_{A}}{d t}=\alpha(1-\delta) E-\gamma_{A} I_{A}-\sigma_{A} I_{A} \\
\frac{d I_{S}}{d t}=\alpha \delta E-\gamma_{S}(1-\theta) I_{S}-\sigma_{S}(1-\theta) I_{S} \\
\frac{d R}{d t}=\gamma_{A} I_{A}+\gamma_{S}(1-\theta) I_{S}+\gamma_{q} I_{q} \\
\frac{d S_{q}}{d t}=\frac{\rho \varphi\left(\beta_{A} \varepsilon E+\beta_{S} \varepsilon E+\beta_{A} I_{A}+\beta_{S} I_{S}\right) S}{N}-\omega E_{q} \\
\frac{d E_{q}}{d t}=\frac{\rho \varphi\left(\beta_{A} \varepsilon E+\beta_{S} \varepsilon E+\beta_{A} I_{A}+\beta_{S} I_{S}\right) S}{N}-\alpha E_{q} \\
\frac{d I_{q}}{d t}=\alpha E_{q}+\theta I_{S}-\gamma_{q} I_{q}-\sigma_{q} I_{q} \\
\frac{d D}{d t}=\sigma_{A} I_{A}+\sigma_{S}(1-\theta) I_{S}+\sigma_{q} I_{q} \\
\frac{d N}{d t}=-\left(\sigma_{A} I_{A}+\sigma_{S}(1-\theta) I_{S}+\sigma_{q} I_{q}\right)
\end{gathered}
$$

Where $\alpha$ is the conversion ratio of latent persons to infected persons, the reciprocal of the incubation period is set at $5.2[24] ; \beta_{A}$ is the probability of asymptomatic infectious in susceptible individuals, with a value of $0.4417[26] ; \beta_{S}$ is the probability of symptomatic infections in the susceptible, with a value of $0.6043[26] ; \gamma_{A}$ is the probability of recovery of asymptomatic infectious people; $\gamma_{S}$ is the probability of recovering from symptomatic infectious; $\gamma_{q}$ is the probability of recovery from quarantined infectious, the values of $\gamma_{A}, \gamma_{S}$ and $\gamma_{q}$ are all $0.1[26] ; \delta_{A}$ is the probability of death from asymptomatic infection[26]; $\delta_{S}$ is the probability of death among the symptomatic infectious[26]; $\delta_{q}$ is the probability of death for the quarantined infectious, the values of $\delta_{A}, \delta_{S}$ and $\delta_{q}$ are all $0.0347[26] ; \varepsilon$ is the probability of lifting the quarantine, the reciprocal of the days of quarantine which refers to $14 ; \theta$ is the probability of the symptomatic infectious being quarantined with a value of $0.8[24] ; \rho$ is the probability 
of quarantine with a value of $0.5[27]$; $\sigma$ is the proportion of symptomatic infectious people with a value of $0.7[24] ; \varphi$ is the influence parameter of environmental factors on COVID-19 transmission, which can be optimized; and $N$ is the total population in each state, which is also a time-dependent parameter.

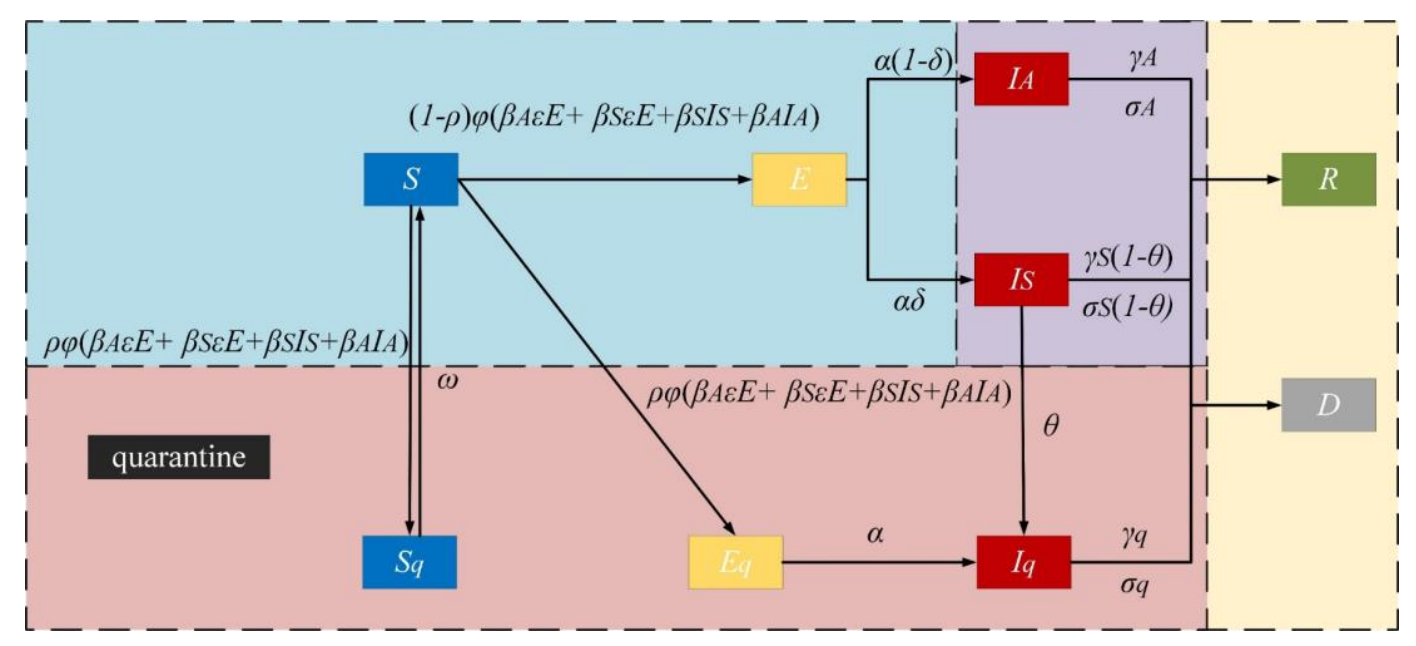

Fig. 3 The improved SEIR dynamic model.

In this study, it appears that the daily active cases that can be obtained by subtracting the cumulative confirmed cases from the cured cases and associated deaths correspond to the quarantined infectious cases in the model. The idea of model fitting is similar to the principle of data assimilation. The least-square method is applied to fit the daily active cases and the quarantined infectious cases simulated by the model so that the effect of environmental factors on COVID-19 $\varphi$ is optimized to be more reasonable. The optimization function used in this study is optim in R software (version 3.6.3), and the optimization method is L-BFGS-B, a type of optimized BFGS, which can increase the box constraint conditions while optimizing and enhancing the function of unconstrained nonlinear programming methods. The machine learning method is used to explore the nonlinear relationship between the optimized $\varphi$ and the dominant 
environmental factors. In this study, a neural network model was established by using the multilayer perceptron in IBM SPSS Statistics 26 to explore the importance of various environmental factors in the response variable $\varphi$.

\section{Results}

The Pearson correlation analysis (Fig. 4a) showed that the morbidity of COVID-19 was significantly correlated with the temperature, relative humidity, population mobility and precipitation $(\mathrm{P}<0.01)$, and the correlation coefficients were $-0.38^{* *}$, $0.534 * *, 0.738 * *$ and $-0.573 * *$, respectively. The wind speed and atmospheric pressure were not significantly associated with morbidity. Therefore, it can be indicated that, to a certain extent, areas with lower temperature, lower humidity and high population mobility are more likely to have higher morbidity from COVID-19, which is a finding that is consistent with those of many studies[28-30]. Due to the significant correlation between precipitation and relative humidity, temperature, relative humidity and population mobility were selected as the dominant environmental factors for further analysis.

The relative risk (RR) of environmental factors corresponds to different lag days (Fig. 4b-d). The results indicated that all the dominant factors had a significant effect on the morbidity of COVID-19. These three dominant factors had a peak value, indicating that there is a relationship between the RR and lag days. For a lag of 6 days, the influence of the temperature on the incidence of COVID-19 reached the highest RR value. For a lag of 2 days, the RR value of the relative humidity reached the highest 
value. For a lag of 1 day, the RR value of population mobility reached the highest. The results demonstrated that socio-ecological factors have a time-lag effect on the incidence of COVID-19, and the RR is different during different lag periods. Compared with meteorological environmental factors, population mobility presents an immediate response to the spread of the COVID-19 pandemic. Accordingly, the lag and cumulative effects of meteorological factors on the spread of infectious diseases are more obvious and favoured by researchers[31-34].
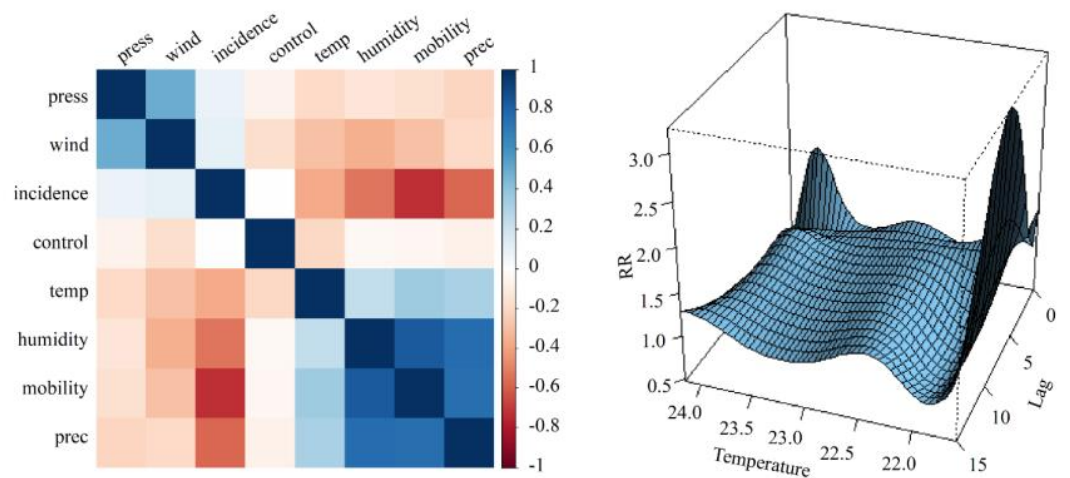

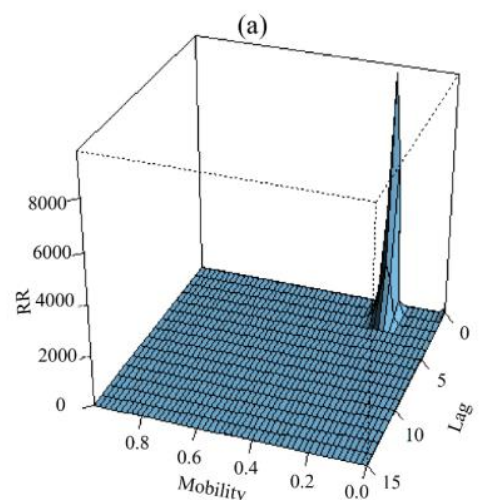

(c)

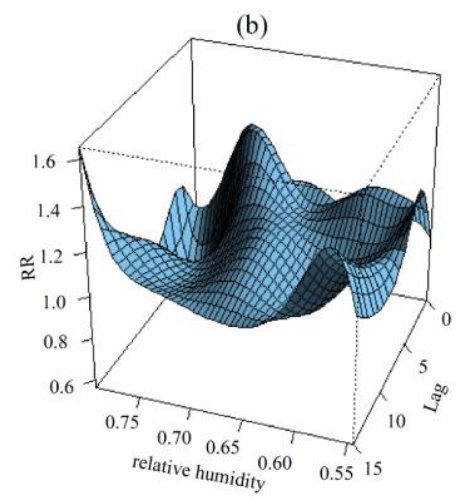

(d)

Fig. 4 Statistical analysis results. (a) The Pearson correlation between environmental factors and incidence of COVID-19. (b)-(d) The RR of COVID-19 as a function of the dominant environmental factors and lag time in 10 Brazilian states. RR, the relative risk. 
The COVID-19 data (from state outbreaks to the end of 2020) of 10 Brazilian states were added to the model for simulation. Fig. 5 shows that the simulation of $I q$ and the actual daily active cases have a strong imitative effect, with $\mathrm{R}^{2}$ values above 0.9, which indicates that the quasi-data assimilation algorithm adopted by the model can better simulate historical data. A multilayer neural network model was built based on $\varphi$ as the output after model optimization and the dominant environmental factors. The results (Fig. 6) demonstrated that the population mobility between regions or within regions caused by human activities played the most significant role in the spread of COVID-19, followed by relative humidity and average daily temperature. Among the factors, population mobility plays a more obvious and important role in the spread of the virus, and the importance of temperature and relative humidity varies in different regions, primarily due to the influence of climatic zones.

Brazil is a country with a very rich climate zone, spanning most climates in the tropics (Fig. 2). The results implied that only the temperature of the Rio de Janeiro and Distrito Federal had a more significant effect on virus transmission than the relative humidity. In other states, relative humidity plays a more important role than temperature. By analysing the climate types of Rio de Janeiro and the Distrito Federal, it can be concluded that both are tropical savanna climates, which will increase in temperature and humidity due to the impact of the Brazil warm current. The annual temperature is generally from $17^{\circ} \mathrm{C}$ to $39^{\circ} \mathrm{C}$, and with a wide range of temperature changes, virus activity can be affected under different conditions, leading to variation 
in the intensity of virus transmission. Overall, relative humidity was more important than the average daily temperature in the model, and the influence of human activities on the transmission of the virus was even more profound. The climate change and ecological environmental change caused by human activities aggravate the emergence and spread of infectious diseases, including COVID-19. In the Amazon rainforest, the Brazilian government has disrupted the environmental protection agencies, allowing the upscaling of illegal forest clearing, which results in the negative consequences of poor social and economic development for indigenous people and the continuous deterioration of their health and sanitary conditions[35-37]. "Destroy ecosystems, especially forest ecosystems, and the virus can move from animals to humans," American science journalist David Quaman explains.
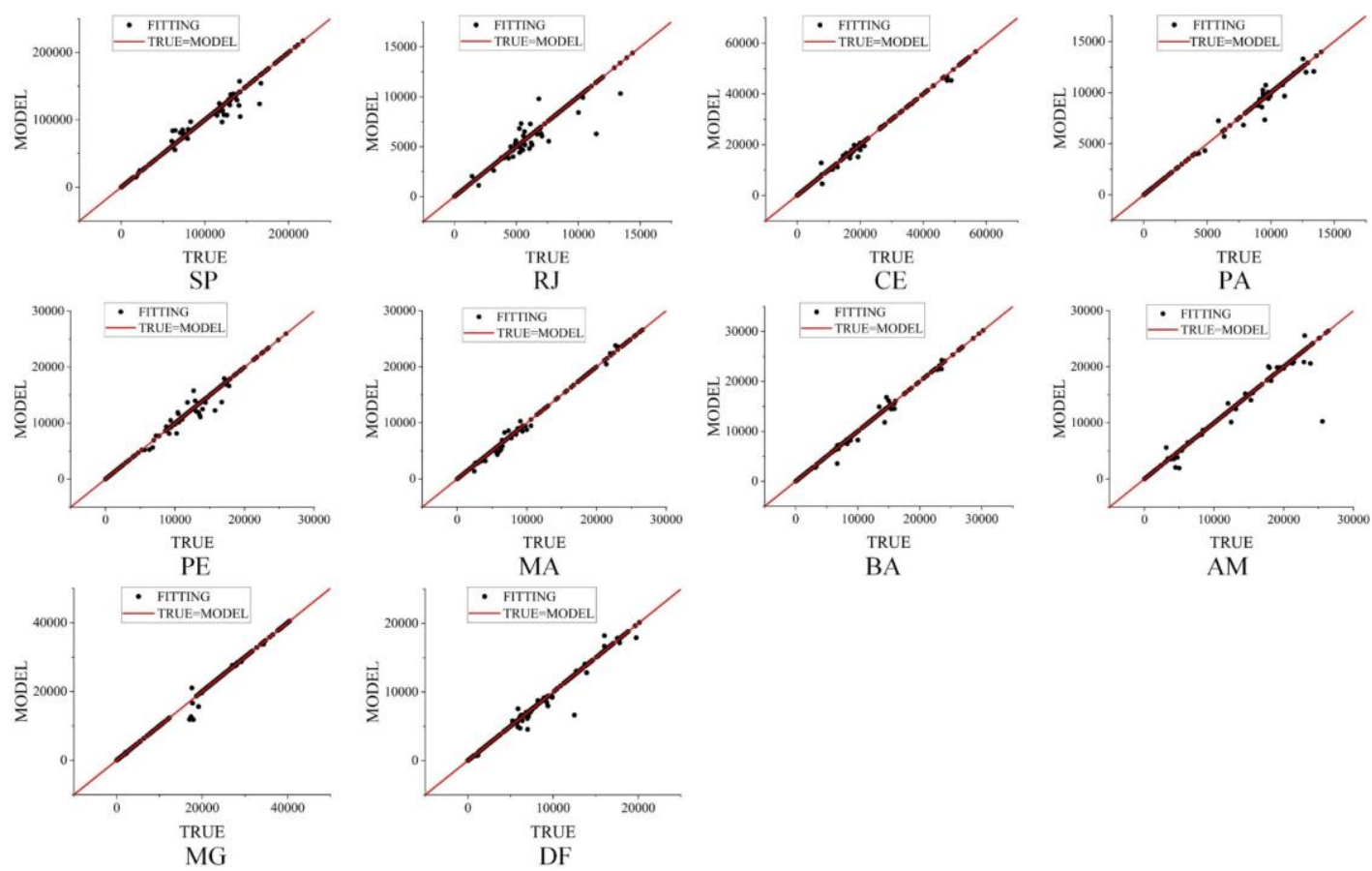

Fig. 5 Results of model fitting. 


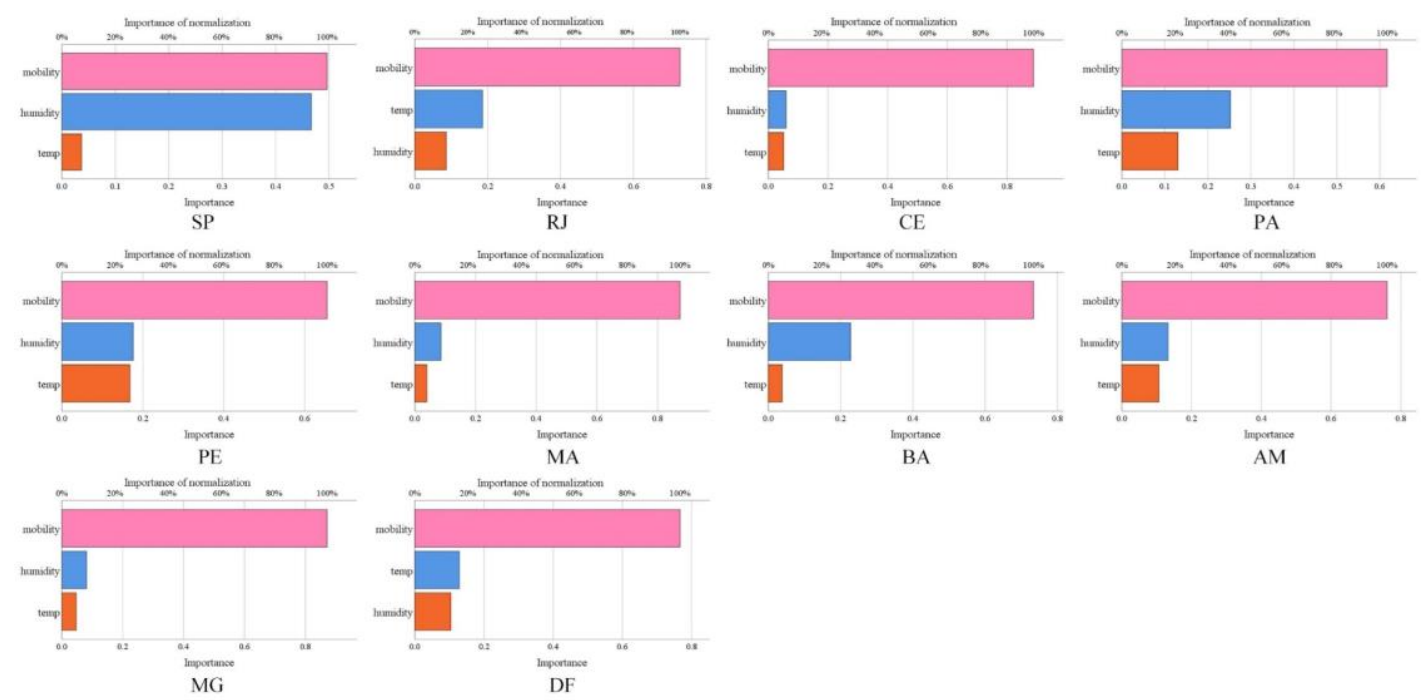

Fig. 6 The importance of dominant environmental factors in the spread of the pandemic.

\section{Discussion}

This study confirmed that environmental factors, including the temperature, relative humidity and population mobility, played an important role in the transmission of COVID-19 in Brazilian states. Compared with social factors, the influence of meteorological factors is low but cannot be ignored, especially temperature and relative humidity. This finding is consistent with other research showing that environmental factors have a time lag effect on the transmission of COVID-19[29, 38], and the hysteresis effect of meteorological factors on human health in the pandemic is more noteworthy because the weather is an uncontrollable variable. In this study, an improved SEIR model was used to analyze the extent to which environmental factors affect the transmission probability of the virus among the population from the perspective of infectious disease dynamics. The influence of different climatic zones in Brazil on COVID-19 transmission was reflected in the regional inconsistency of the 
temperature and relative humidity effects on COVID-19 transmission. The impact of different climate patterns on the pandemic in Brazil is worth exploring.

This study proposed an improved SEIR model in which the impact of socioecological factors was added as an environmental propagation probability for the first time. By using an improved SEIR model, the study explored the influence of the environment on the transmission of COVID-19 from the perspective of system dynamics. Environmental factors further influence the process of virus transmission by affecting the probability of virus transmission among people. In addition, this study proposed using a machine learning method to establish the nonlinear relationship between socio-ecological factors and environmental propagation probability, and the current scientific research method was fully applied in this study. The organic combination of infectious disease models and machine learning methods to explore the interconnection between environmental factors and virus transmission provides an important methodological reference.

Although some important conclusions have been obtained by applying the improved SEIR model, there are also limitations. First, environmental factors were considered in ways that were not comprehensive. Other factors such as air pollution, biodiversity, virus variations, and population immunity were not considered, but the factors considered at present are relatively adequate. Second, the improved SEIR model regards the impact of environmental factors on the COVID-19 pandemic as a comprehensive time-varying parameter and does not reflect the individual impact 
effectiveness of each factor in the improved SEIR model. However, this study uses a machine learning method to establish the nonlinear relationship between environmental factors and environmental propagation probability $\varphi$, which is also a sign of progress and can be referenced in the future. The results of this paper provide many instructions for the prevention and control of COVID-19. Appropriately reducing population mobility between regions in the cold and dry seasons and emphasizing personal hygiene are effective measures of relieving the spread of the pandemic. In states such as Rio de Janeiro and the Distrito Federal, special attention should be given to the untoward effect of temperature changes on COVID-19 transmission, while other regions should pay attention to the effect of humidity changes during the alternate periods of dry and wet conditions.

\section{Conclusion}

In summary, this study argued that changes in environmental factors can affect the transmission of COVID-19, and compared with social factors, the change in natural factors has a small but significant impact on the transmission process. The present findings confirmed that environmental factors influence human risk by influencing the probability of viral transmission among people, and the environmental propagation probability is insignificant but not negligible. As influenced by climatic zones, meteorological factors showed heterogeneity in COVID-19 transmission in different regions. The quest to explore the causal relationship between socio-ecological factors 
and COVID-19 continues to be a great impetus for researchers to discover and optimize new approaches.

\section{Acknowledgements}

This research was funded by the Tang Scholar Program (K. Jia is a Tang Scholar of Beijing Normal University).

\section{Author contributions}

Conceptualization: Kun Jia, Wenwu Zhao; Methodology: Jie Li, Kun Jia; Formal analysis and investigation: Jie Li, Kun Jia, Bo Yuan; Writing - original draft preparation: Jie Li; Writing - review and editing: Kun Jia, Wenwu Zhao, Bo Yuan, Yanxu Liu; Funding acquisition: Kun Jia, Wenwu Zhao; Resources: Jie Li, Bo Yuan, Kun Jia, Wenwu Zhao; Supervision: Kun Jia, Wenwu Zhao.

\section{Data availability}

The datasets generated during the current study are available from the corresponding author on reasonable request.

\section{Declaration of competing interest}

The authors declare that they have no known competing financial interests or personal relationships that could have appeared to influence the work reported in this paper.

\section{References:}

1. Lopes, M.F.: From denial to hope: Brazil deals with a prolonged COVID-19 epidemic course. Nat. Immunol. 22, 256-257 (2021)

2. Neto, O.P., Kennedy, D.M., Reis, J.C., Wang, Y., Brizzi, A.C.B., Zambrano, G.J., de Souza, J.M., Pedroso, W., de Mello Pedreiro, R.C., de Matos Brizzi, B.: Mathematical model of COVID19 intervention scenarios for São Paulo—Brazil. Nat. Commun. 12, 1-13 (2021)

3. Fernandes, J.S.C., da Silva, R.S., Silva, A.C., Villela, D.C., Mendonça, V.A., Lacerda, A.C.R.: Altitude conditions seem to determine the evolution of COVID-19 in Brazil. Sci. Rep. 11, 1-12 
4. Costello, A., Abbas, M., Allen, A., Ball, S., Bell, S., Bellamy, R., Friel, S., Groce, N., Johnson, A., Kett, M.: Managing the health effects of climate change: lancet and University College London Institute for Global Health Commission. Lancet. 373, 1693-1733 (2009)

5. Liang, L., Gong, P.: Climate change and human infectious diseases: A synthesis of research findings from global and spatio-temporal perspectives. Environ. Int. 103, 99-108 (2017)

6. McMichael, A.J., Woodruff, R.E., Hales, S.: Climate change and human health: present and future risks. Lancet. 367, 859-869 (2006)

7. Watts, N., Adger, W.N., Agnolucci, P., Blackstock, J., Byass, P., Cai, W., Chaytor, S., Colbourn, T., Collins, M., Cooper, A.: Health and climate change: policy responses to protect public health. Lancet. 386, 1861-1914 (2015)

8. Franch-Pardo, I., Napoletano, B.M., Rosete-Verges, F., Billa, L.: Spatial analysis and GIS in the study of COVID-19. A review. Sci. Total Environ. 739, 140033 (2020)

9. Gupta, S., Raghuwanshi, G.S., Chanda, A.: Effect of weather on COVID-19 spread in the US: A prediction model for India in 2020. Sci. Total Environ. 728, 138860 (2020)

10. Ujiie, M., Tsuzuki, S., Ohmagari, N.: Effect of temperature on the infectivity of COVID-19. Int. J. Infect. Dis. 95, 301-303 (2020)

11. Jia, J.S., Lu, X., Yuan, Y., Xu, G., Jia, J., Christakis, N.A.: Population flow drives spatiotemporal distribution of COVID-19 in China. Nature. 582, 389-394 (2020)

12. Nouvellet, P., Bhatia, S., Cori, A., Ainslie, K.E.C., Baguelin, M., Bhatt, S., Boonyasiri, A., Brazeau, N.F., Cattarino, L., Cooper, L. V: Reduction in mobility and COVID-19 transmission. Nat. Commun. 12, 1-9 (2021)

13. Cooper, I., Mondal, A., Antonopoulos, C.G.: A SIR model assumption for the spread of COVID19 in different communities. Chaos, Solitons \& Fractals. 139, 110057 (2020)

14. Grimm, V., Mengel, F., Schmidt, M.: Extensions of the SEIR model for the analysis of tailored social distancing and tracing approaches to cope with COVID-19. Sci. Rep. 11, 1-16 (2021)

15. Liu, F., Wang, J., Liu, J., Li, Y., Liu, D., Tong, J., Li, Z., Yu, D., Fan, Y., Bi, X.: Predicting and analyzing the COVID-19 epidemic in China: Based on SEIRD, LSTM and GWR models. PLoS One. $15, \mathrm{e} 0238280(2020)$ 
16. López, L., Rodo, X.: A modified SEIR model to predict the COVID-19 outbreak in Spain and Italy: simulating control scenarios and multi-scale epidemics. Results Phys. 21, 103746 (2021)

17. Mwalili, S., Kimathi, M., Ojiambo, V., Gathungu, D., Mbogo, R.: SEIR model for COVID-19 dynamics incorporating the environment and social distancing. BMC Res. Notes. 13, 1-5 (2020)

18. Alvares, C.A., Stape, J.L., Sentelhas, P.C., Gonçalves, J.L. de M., Sparovek, G.: Köppen’s climate classification map for Brazil. Meteorol. Zeitschrift. 22, 711-728 (2013)

19. Gasparrini, A., Leone, M.: Attributable risk from distributed lag models. BMC Med. Res. Methodol. 14, 1-8 (2014)

20. Fu, S., Wang, B., Zhou, J., Xu, X., Liu, J., Ma, Y., Li, L., He, X., Li, S., Niu, J.: Meteorological factors, governmental responses and COVID-19: Evidence from four European countries. Environ. Res. 194, 110596 (2021)

21. Runkle, J.D., Sugg, M.M., Leeper, R.D., Rao, Y., Matthews, J.L., Rennie, J.J.: Short-term effects of specific humidity and temperature on COVID-19 morbidity in select US cities. Sci. Total Environ. 740, 140093 (2020)

22. Shi, P., Dong, Y., Yan, H., Zhao, C., Li, X., Liu, W., He, M., Tang, S., Xi, S.: Impact of temperature on the dynamics of the COVID-19 outbreak in China. Sci. Total Environ. 728, $138890(2020)$

23. Li, M.Y., Muldowney, J.S.: Global stability for the SEIR model in epidemiology. Math. Biosci. $125,155-164(1995)$

24. Wei, Y.Y., Lu, Z.Z., Du, Z.C., Zhang, Z.J., Zhao, Y., Shen, S.P., Wang, B., Hao, Y.T., Chen, F.: Fitting and forecasting the trend of COVID-19 by SEIR (+ CAQ) dynamic model. Zhonghua liu xing bing xue za zhi= Zhonghua liuxingbingxue zazhi. 41, 470-475 (2020)

25. Cao, S., Feng, P., Shi, P.: Study on the epidemic development of COVID-19 in Hubei province by a modified SEIR model. J. Zhejiang Univ. (Medical Sci. 49, 178-184 (2020)

26. Bastos, S.B., Cajueiro, D.O.: Modeling and forecasting the early evolution of the Covid-19 pandemic in Brazil. Sci. Rep. 10, 1-10 (2020) 
27. Wu, J.T., Leung, K., Leung, G.M.: Nowcasting and forecasting the potential domestic and international spread of the 2019-nCoV outbreak originating in Wuhan, China: a modelling study. Lancet. 395, 689-697 (2020)

28. Guo, X.-J., Zhang, H., Zeng, Y.-P.: Transmissibility of COVID-19 and its association with temperature and humidity. (2020)

29. Ma, Y., Zhao, Y., Liu, J., He, X., Wang, B., Fu, S., Yan, J., Niu, J., Zhou, J., Luo, B.: Effects of temperature variation and humidity on the death of COVID-19 in Wuhan, China. Sci. Total Environ. 724, 138226 (2020)

30. Ozyigit, A.: Understanding Covid-19 transmission: The effect of temperature and health behavior on transmission rates. Infect. Dis. Heal. 25, 233-238 (2020)

31. Chien, L.-C., Yu, H.-L.: Impact of meteorological factors on the spatiotemporal patterns of dengue fever incidence. Environ. Int. 73, 46-56 (2014)

32. Fang, X., Ai, J., Liu, W., Ji, H., Zhang, X., Peng, Z., Wu, Y., Shi, Y., Shen, W., Bao, C.: Epidemiology of infectious diarrhoea and the relationship with etiological and meteorological factors in Jiangsu Province, China. Sci. Rep. 9, 1-9 (2019)

33. Li, Z., Wang, L., Sun, W., Hou, X., Yang, H., Sun, L., Xu, S., Sun, Q., Zhang, J., Song, H.: Identifying high-risk areas of bacillary dysentery and associated meteorological factors in Wuhan, China. Sci. Rep. 3, 1-6 (2013)

34. Xiao, Y., He, L., Chen, Y., Wang, Q., Meng, Q., Chang, W., Xiong, L., Yu, Z.: The influence of meteorological factors on tuberculosis incidence in Southwest China from 2006 to 2015. Sci. Rep. 8, 1-8 (2018)

35. Pontes, M.R.N., Lima, J.P.: Brazil’s COVID-19 response. Lancet. 396, e34 (2020)

36. Ferrante, L., Fearnside, P.M.: Brazil threatens Indigenous lands. Science (80-. ). 368, 481-482 (2020)

37. Vale, M.M., Berenguer, E., de Menezes, M.A., de Castro, E.B.V., de Siqueira, L.P., Rita de Cássia, Q.P.: The COVID-19 pandemic as an opportunity to weaken environmental protection in Brazil. Biol. Conserv. 255, 108994 (2021) 
38. Liu, J., Zhou, J., Yao, J., Zhang, X., Li, L., Xu, X., He, X., Wang, B., Fu, S., Niu, T.: Impact of meteorological factors on the COVID-19 transmission: A multi-city study in China. Sci. Total Environ. 726, 138513 (2020) 


\section{Figures}

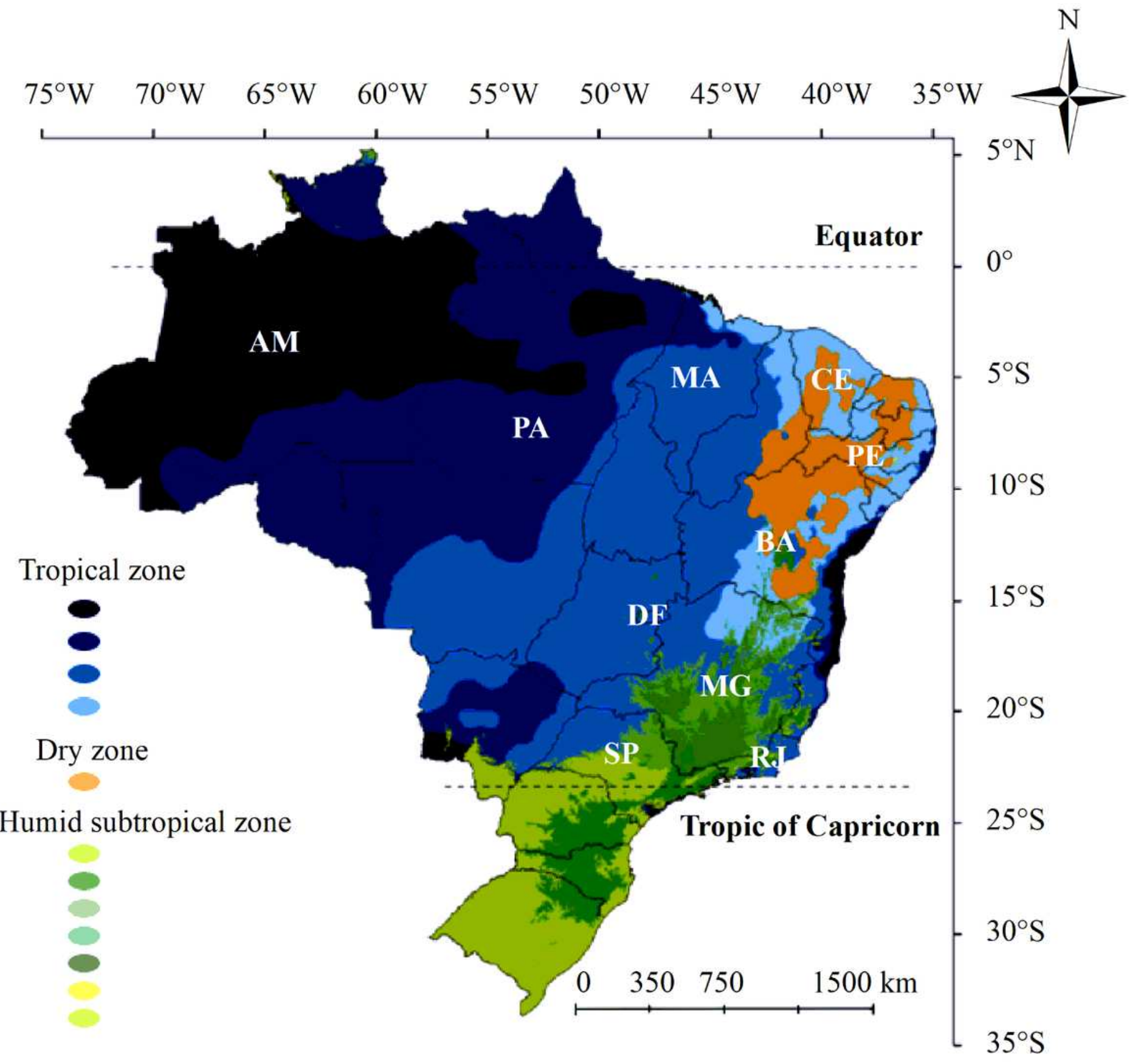

\section{Figure 1}

The location of Brazil with its Köppen climate types. Note: The designations employed and the presentation of the material on this map do not imply the expression of any opinion whatsoever on the part of Research Square concerning the legal status of any country, territory, city or area or of its authorities, or concerning the delimitation of its frontiers or boundaries. This map has been provided by the authors. 

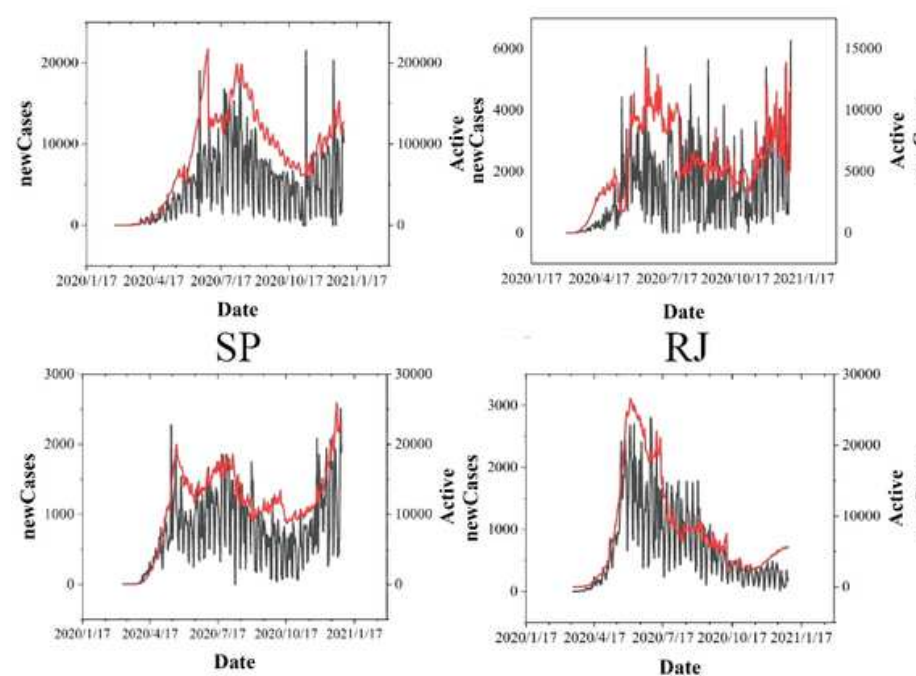

PE
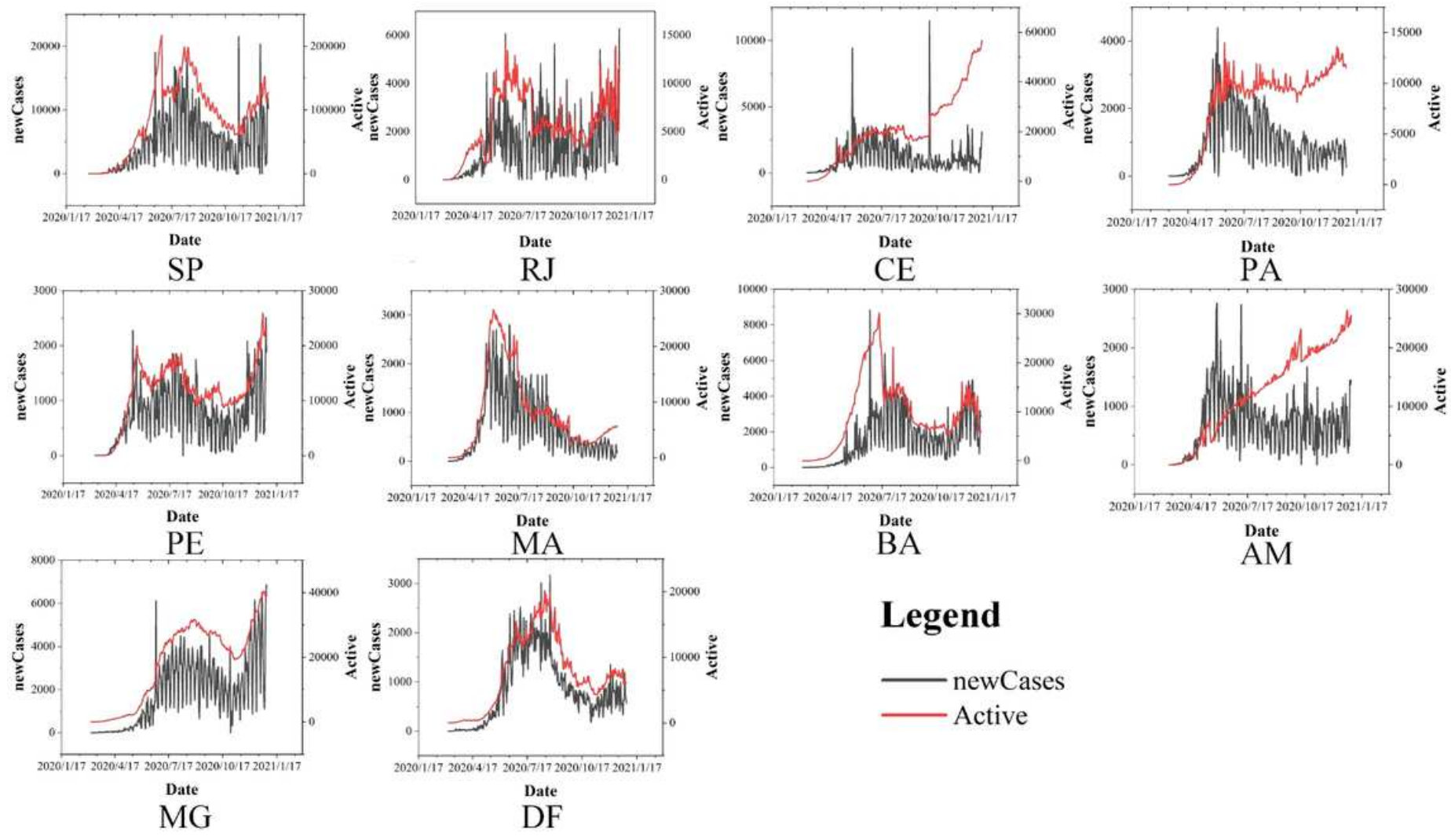

\section{Legend}

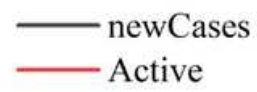

Figure 2

COVID-19 data from 10 states in Brazil.

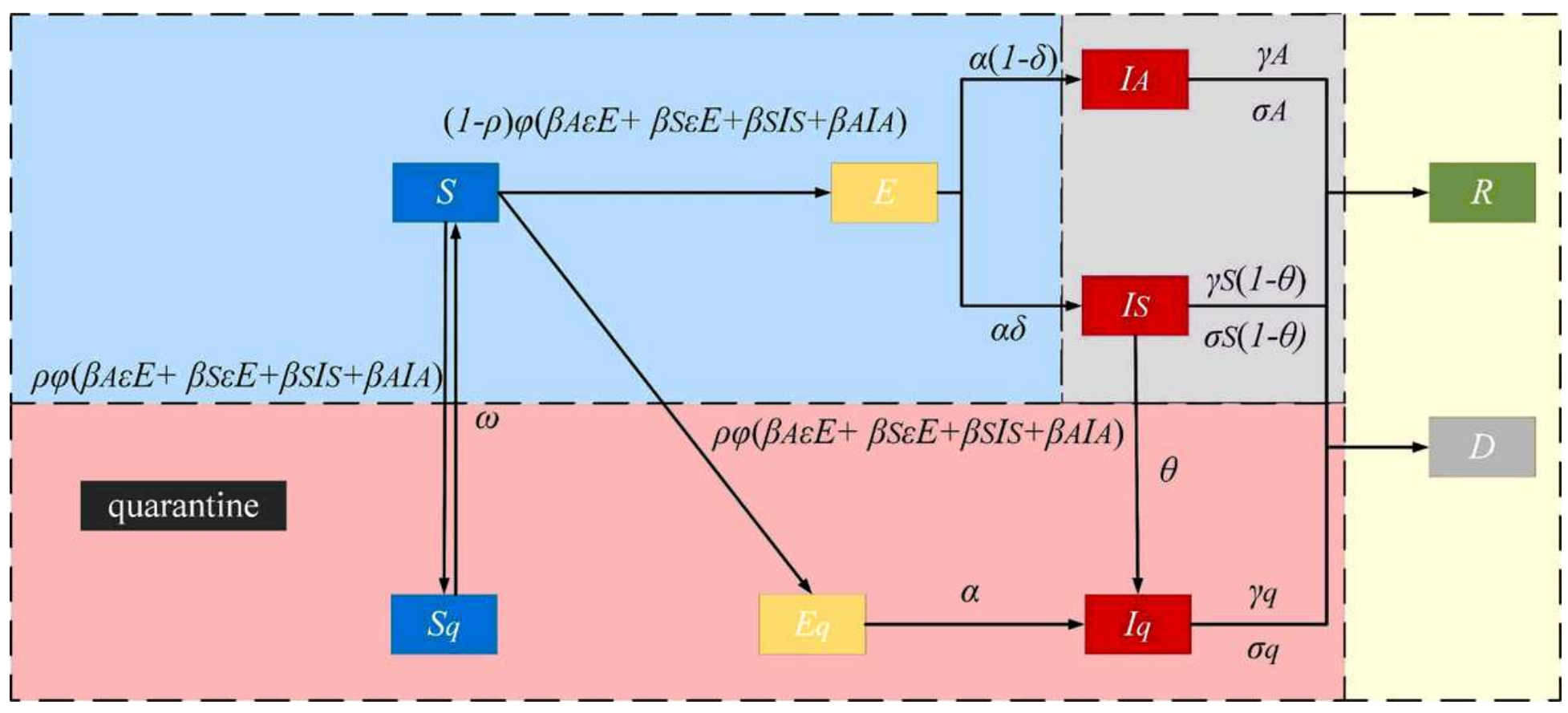

Figure 3

The improved SEIR dynamic model. 


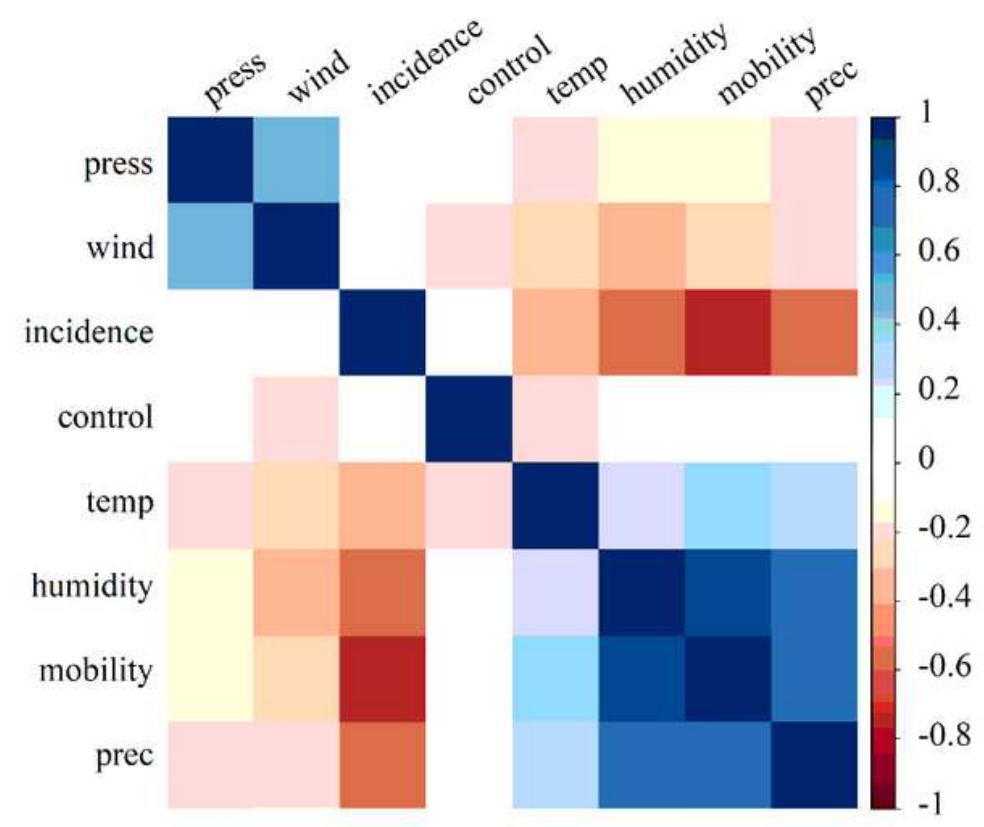

(a)

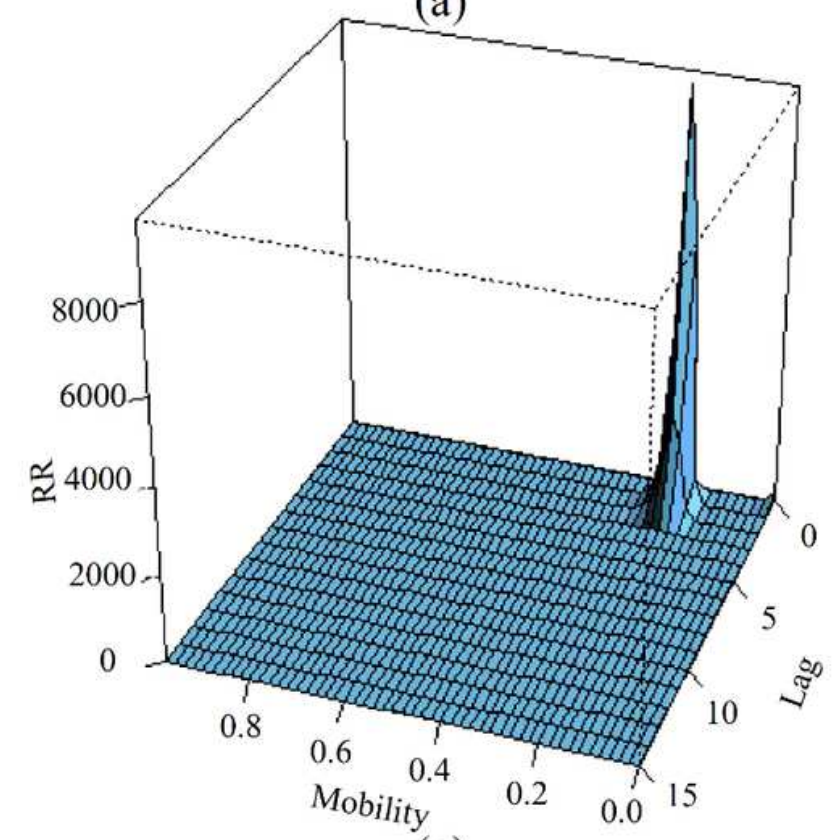

(c)

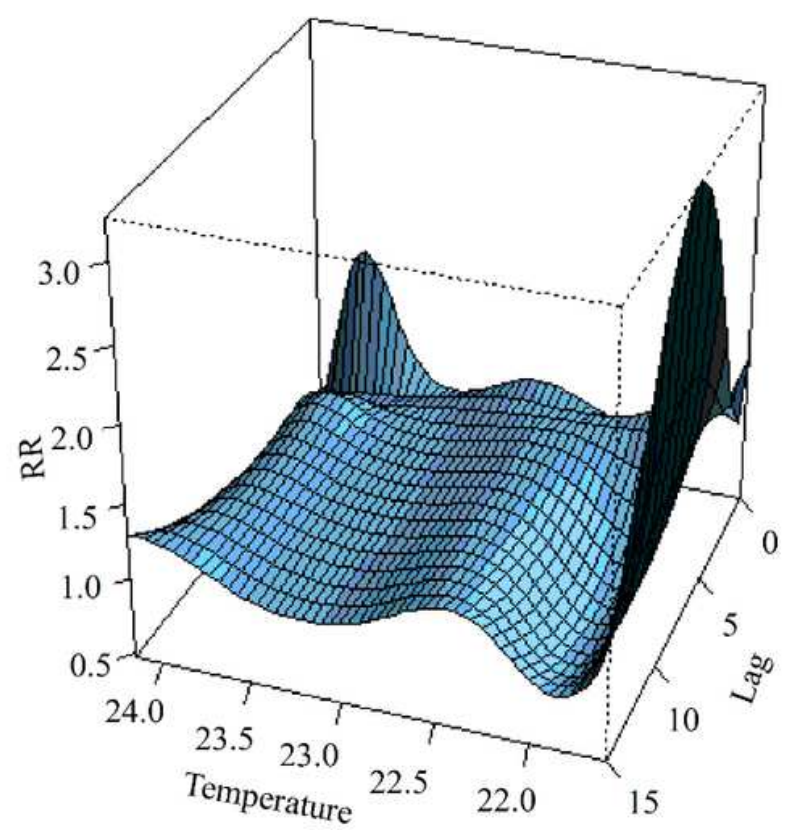

(b)

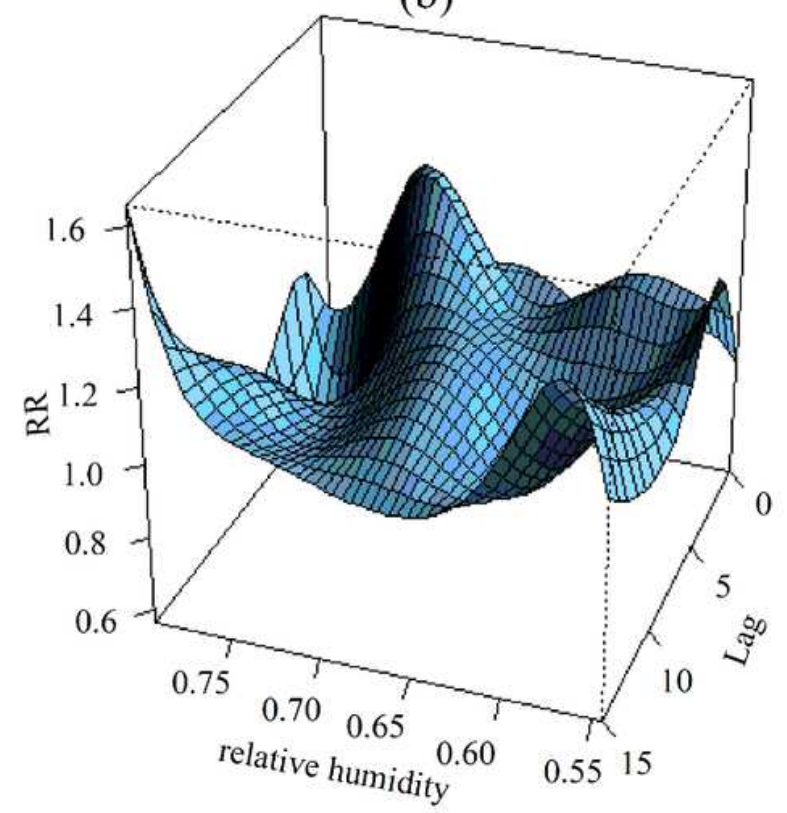

(d)

\section{Figure 4}

Statistical analysis results. (a) The Pearson correlation between environmental factors and incidence of COVID-19. (b)-(d) The RR of COVID-19 as a function of the dominant environmental factors and lag time in 10 Brazilian states. RR, the relative risk. 


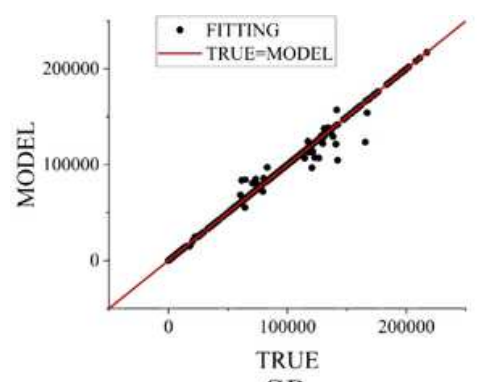

SP

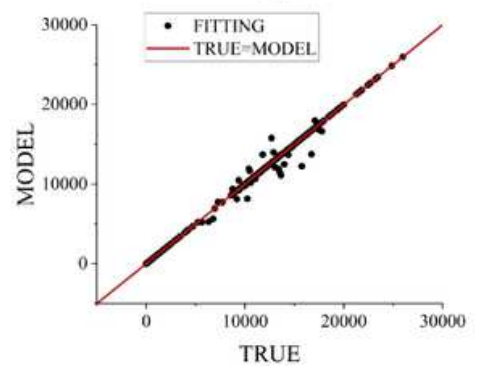

PE

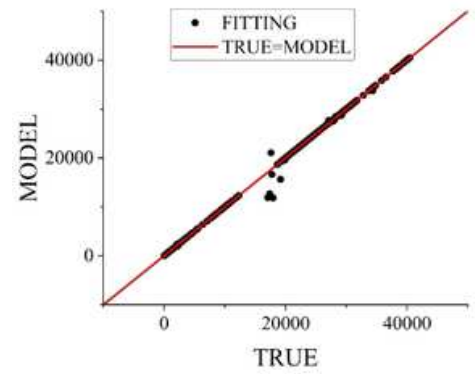

MG

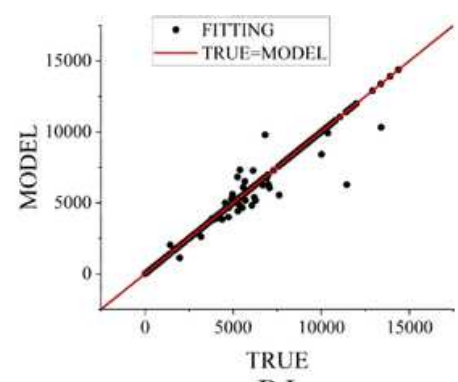

RJ

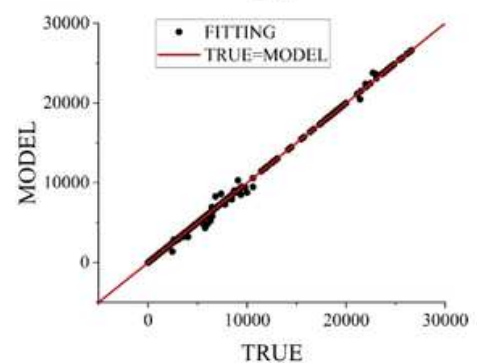

MA

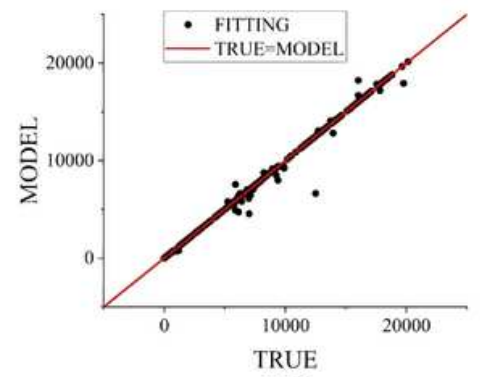

DF

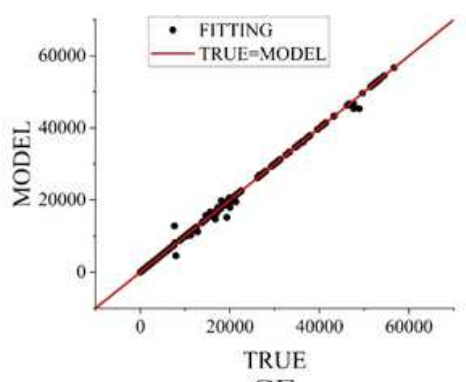

CE

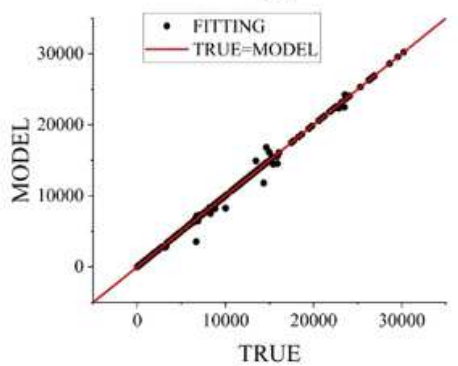

BA
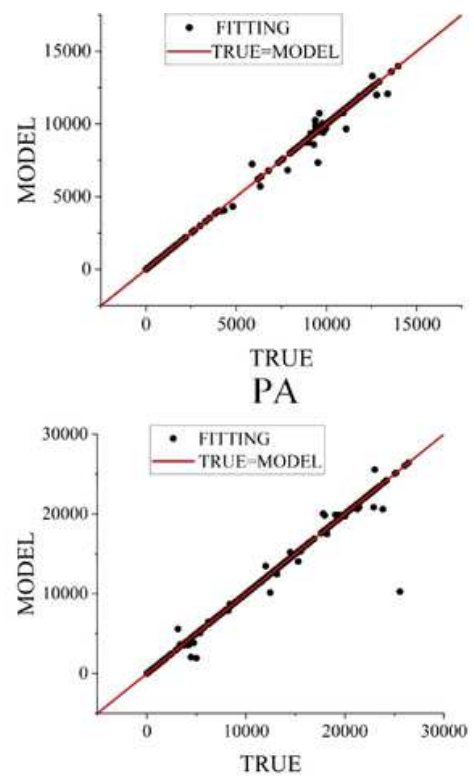

AM

\section{Figure 5}

Results of model fitting.
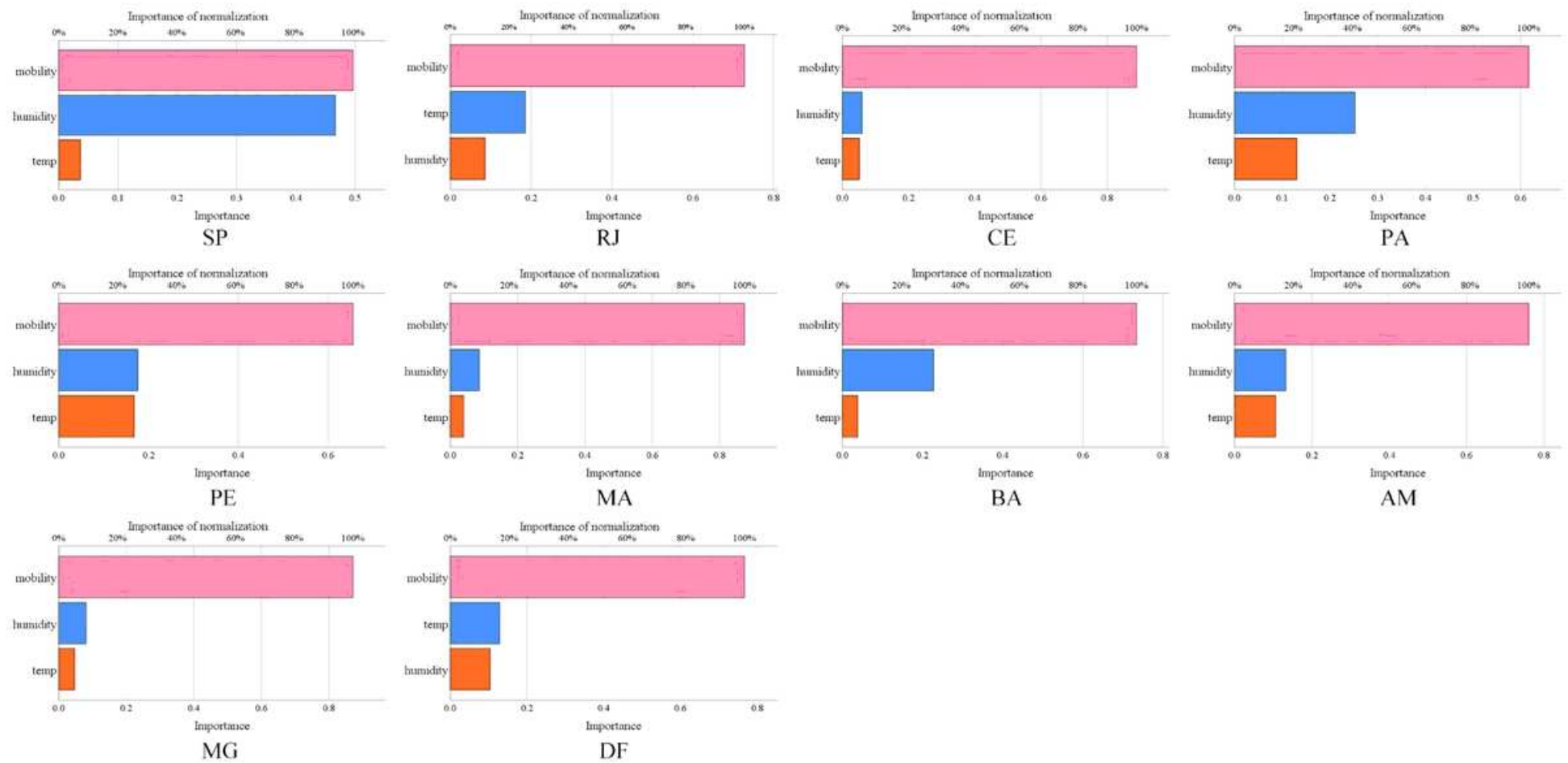
Figure 6

The importance of dominant environmental factors in the spread of the pandemic. 